\title{
Letter
}

\section{Editorial Retraction}

\section{Donald Kennedy}

American Association for the Advancement of Science, 1200 New York Avenue NW, Washington, D.C. 20005, USA.*

*To whom correspondence should be addressed. E-mail: dkennedy@aaas.org

The final report from the Investigation Committee of Seoul National University (SNU) (1) has concluded that the authors of two papers published in Science $(2,3)$ have engaged in research misconduct and that the papers contain fabricated data. With regard to Hwang et al., 2004 (2), the Investigation Committee reported that the data showing that DNA from human embryonic stem cell line NT-1 is identical to that of the donor are invalid because they are the result of fabrication, as is the evidence that NT-1 is a bona fide stem cell line. Further, the committee found that the claim in Hwang et al., 2005 (3) that 11 patient-specific embryonic stem cells line were derived from cloned blastocysts is based on fabricated data. According to the report of the Investigation Committee, the laboratory "does not possess patient-specific stem cell lines or any scientific basis for claiming to have created one.” Because the final report of the SNU investigation indicated that a significant amount of the data presented in both papers is fabricated, the editors of Science feel that an immediate and unconditional retraction of both papers is needed. We therefore retract these two papers and advise the scientific community that the results reported in them are deemed to be invalid.

As we post this retraction, six of the 15 authors of Hwang et al., 2004 (2) have agreed to retract their paper. All of the 25 authors of Hwang et al., 2005 (3) have agreed to retract their paper.

Science regrets the time that the peer reviewers and others spent evaluating these papers as well as the time and resources that the scientific community may have spent trying to replicate these results.

\section{Donald Kennedy}

Editor-in-Chief

\section{References}

1. Investigation Committee Report, Seoul National University, 10 Jan. 2006. (Members: Chairman Myung-Hee Chung, SNU, Uhtaek Oh, SNU, Hong-Hee Kim, SNU, Un Jong Pak, SNU, Yong Sung Lee, Hanyang University, In Won Lee, SNU, In Kwon Chung, Yonsei University, Jin Ho Chung, SNU)

2. W.-S. Hwang et al., Evidence of A Pluripotent Human Embryonic Stem Cell Line Derived From a Cloned Blastocyst, Science 303, 1669 (2004).

3. W.-S. Hwang et al., Patient-Specific Embryonic Stem Cells Derived from Human SCNT Blastocysts, Science 308, 1777 (2005)

Published online 12 January 2006; 10.1126/science.1124926 Include this information when citing this paper. 